\section{Smear negative pulmonary tuberculosis and infectivity}

\section{Thapa B}

Kathmandu Medical College and Teaching Hospital, Genesis Laboratory and Research, Kathmandu, Nepal

Dr. Badri Thapa, email: badri_bishal@yahoo.com, Tel. No.: (+97)-9841220108
The smear negative tuberculosis (TB) patients (Pulmonary (PTB), Extra pulmonary (EPPTB)) are infectious and transmission contributes to $13 \%$ to $41 \%$ depending upon the settings and the type of patients.

The TB patients should have at least 5,000-10,000 bacilli in sputum for detection by smear microscopy. In contrast, patients whose bacillary load is less than the mentioned number are not detected by the smear microscopy. ${ }^{1}$ Smear positive patients spreads the infections to others and TB control programmes are aggressive in early diagnosis of smear positive TB patients. It has been believed that smear positive patients spreads the disease and smear negative cases are less infectious than the smear positive cases and do not contribute for transmission. However there are sufficient evidences to show that the smear negative tuberculosis can also transmit the disease.

A study from San Fransisco has showed that at least $17 \%$ of the TB transmission is contributed by smear negative TB cases. $^{2}$ A molecular epidemiological study was conducted in two groups (Group 1, PTB or PTB + EPTB; Group 2, Group 1 + EPTB) in Vancouver General Hospital to trace the transmission chain of smear negative tuberculosis. ${ }^{1}$ It revealed that the proportion of episodes of transmission was $17 \%$ to $21 \%$ in Group 1 and 25\% to 41\% in Group 2. The result showed that the smear negative TB transmission accounted for at least one sixth of the total culture positive TB cases. This study has also highlighted that the transmission is also possible through the EPTB cases.

In a study, a infant having skin lesion and scarring of the lung apical region in the radiograph was smear negative. ${ }^{3}$ The AFB was demonstrated from the skin lesion. The man with the similar clinical presentations used to take care of the infant for several hours a day. The genotypes from the infant and the man matched perfectly by IS6110 RFLP technique. This further illustrates the infectiousness of the smear negative tuberculosis. Similarly, a study has reported the extensive transmission of the tuberculosis in school in Luton, England from a child with smear negative pulmonary tuberculosis. ${ }^{4}$ The index case was a 9 year old boy of black African origin born in United Kingdom. A study in Netherland has revealed that $12.6 \%$ of the tuberculosis transmission in 394 
clusters (total of 1285 patients) was attributable to the transmission from a patient with smear negative tuberculosis. ${ }^{5}$

The bacillary burden in smear negative PTB are less but they have been reported with different clinical and radiographic findings than those with smear positives. ${ }^{6}$ Smear negatives and culture positives were at equal odds of having tuberculin test positive, higher odds of having HIV sero-positive and mediastinal lymadenopathy.

\section{REFERENCES}

1. Hernandez-Garduno E, Cook V, Kunimoto D, et al. Transmission of tuberculosis from smear negative patients: a molecular epidemiology study. Thorax 2004; 59:286-290.

2. Ber MA, Warren SA Salamon, et al. Transmission of Mycobacterium tuberculosis from patients smearnegative for acid fast bacilli. Lancet 1999; 353: 444-449.

3. Jason E, John J, Carol D. Tuberculosis transmission from a patient with skin lesions and a negative sputum smear. New England J Med 2004: 350: 2527-2528.

4. Paranjothy S, Eisenhut M, Lilley $M$ et al. Extensive transmission of Mycoabcterium tuberculosis from 9 year old child with pulmonary tuberculosis and negative sputum smear. British Med J 2008. DOI:http://dx.doi.org /10.1136/bmj.a1184

5. Tostman A, Kik SV, Kalisvaart, et al. Tuberculosis transmission by patients with smear negative pulmonary tuberculosis in a large cohort in the Netherlands. Clin Infec Dis 2008; 47: 1135-1142.

6. Kanaya AM, Glidden DV, Chamber HF. Idenifying pulmonary tuberculosis in patients with negative sputum smear results. Chest 2001; 120: 349-355. 